\title{
Histoires doubles : quand les écrivains s'illustrent
}

\section{Serge Linarès}

\section{OpenEdition}

Journals

Édition électronique

URL : http://journals.openedition.org/edl/570

DOI : $10.4000 /$ edl. 570

ISSN : 2296-5084

\section{Éditeur}

Université de Lausanne

\section{Édition imprimée}

Date de publication : 15 décembre 2013

Pagination : 15-44

ISBN : $978-2-940331-33-8$

ISSN : 0014-2026

\section{Référence électronique}

Serge Linarès, " Histoires doubles : quand les écrivains s'illustrent », Études de lettres [En ligne], 3-4 | 2013, mis en ligne le 15 décembre 2016, consulté le 18 décembre 2020. URL : http:// journals.openedition.org/edl/570 ; DOl : https://doi.org/10.4000/edl.570 


\section{HISTOIRES DOUBLES : QUAND LES ÉCRIVAINS S'ILLUSTRENT}

En illustrant leurs œuvres narratives, les écrivains artistes tendent souvent à explorer l'iconicité scripturale. Lewis Carroll donne ainsi tous ses soins à l'objet livre et au tracé des lettres dans sa version imagée des Aventures d'Alice au cour de la terre, et Kipling s'enquiert des origines de l'écriture dans les dessins des Histoires comme çà. Reste que l'antériorité éventuelle de l'image sur l'énoncé précipite davantage les écrivains dans une logique visuelle qui, sans invalider la narrativité, la soumet au régime de la spatialité et de la figuration. La spatialité conditionne alors les modes d'apparition de l'iconographie comme du texte. Et la figuration prête parfois à tous les vertiges de la représentation, notamment de soi : ainsi chez Musset (Le Mariage de Pauline Garcia avec Louis Viardot) et chez Cocteau (Album des Eugènes). La narration, en sa qualité de médiatrice entre image et langage, transcende toute valeur esthétique et se fait porteuse d'une tentative pour questionner, sinon fixer, l'identité.

Tendre sa réflexion vers les pratiques doubles de certains écrivains, lorsqu'il s'agit de questionner les croisements du textuel et du visuel dans les livres à figures, c'est d'abord restreindre le champ d'étude à des cas d'espèce, par comparaison avec l'énorme production à deux mains des ouvrages illustrés. C'est ensuite risquer d'épointer l'acuité de la problématique, que pourrait bien neutraliser la dimension apparemment périphérique et récréative des compléments graphiques apportés par les auteurs à leurs écrits. S'il n'y a pas lieu de majorer l'importance du corpus considéré, largement minoritaire en termes d'occurrences, ce dernier nous incline pourtant à examiner jusque dans ses replis et dans ses perspectives le transfert du verbal au figural. L'expression plastique des écrivains peut, en effet, se hausser au niveau d'une interrogation cruciale sur les enjeux de l'articulation entre les langages littéraire et artistique. 
A cela une raison principale: la création iconique, loin de relever de l'évidence, a consisté, pour les auteurs, en une conquête progressive depuis le Romantisme, gagnée sur l'obligation à la mimèsis, les astreintes de la rhétorique, la hiérarchie du paragone, le cantonnement du lyrisme, voire sur la différenciation sémiotique de la poésie et des arts de l'espace, formalisée par Lessing dans son Laocoon. Il est vrai que l'illustration fournit à nombre d'écrivains l'occasion d'entrer dans la sphère du visible sans se désorbiter de l'attraction linguistique. Elle dépose de la validité d'une expérience du dessin, mais ne compromet guère le maintien de la régulation langagière dans l'action du scripteur qui goûte à l'emploi de la ligne et de la couleur, sous le contrôle de l'énoncé. Jusqu'à la fin du $\mathrm{XIX}^{\mathrm{e}}$ siècle pour le moins, la domination du logos s'impose généralement à l'apparition des images dans le livre (surtout d'obédience narrative), et détermine leur degré de conformité à la formulation des descriptions et des péripéties. Pour autant, on veut croire à l'amorce de cette réflexion que, n'étant artistes de vocation ni de métier, bien des hommes de lettres s'essaient à orner de représentations certains récits dans un esprit qui doit peu à celui des artistes placés dans une situation identique. S'ils s'engagent dans la voie de la conversion visuelle à proximité du médium d'origine, il y a fort à parier que leurs intentions, voire les fruits de leur changement de moyen expressif, ne sont pas rabattables sur ceux des illustrateurs de carrière et singularisent leurs positions parmi les artisans d'images. A l'appui d'une pareille hypothèse, opter exclusivement pour un corpus d'œuvres narratives pourrait réduire les possibilités de distinguer l'originalité des écrivains artistes, car la dépendance de l'iconographie à la rédaction s'y révèle par tradition plus marquée que pour d'autres genres, au premier chef la poésie. Comme ces illustrateurs amateurs sont alors au plus près de leur domaine d'extraction et que leurs homologues professionnels prouvent volontiers leur fidélité aux textes qu'ils visualisent, le risque est grand qu'un tel vivier d'intrigues figurées laisse simplement au lecteur des sentiments d'initiative gratuite et de maladresse technique. Sans demeurer sous l'impression d'avoir affaire à des tentatives artistiques dépourvues de profondeur, sans entrer non plus dans des considérations esthétiques, du ressort de chacun, il nous appartiendra de montrer que la coexistence du lisible et du visible en mode narratif prend des contours spécifiques sous la gouverne d'écrivains en quête d'ouverture transmédiale, y compris dans les formes les moins considérées, notamment les ouvrages pour la jeunesse. Il est, sur ce chapitre, de toute 
Fig. 1 - Victor Hugo, Victor Hugo 1866, Les Travailleurs de la mer.

impossibilité de prétendre à l'exhaustivité. Aussi partiel et partial que soit notre choix d'auteurs, il entend toutefois explorer, dans ses grandes largeurs, l'étendue des échanges entre le récit et l'image, selon qu'il s'agit de livres illustrés ou, moins communément, d'histoires mixtes.

Rares sont les écrivains qui, avant le $\mathrm{XX}^{\mathrm{e}}$ siècle, parviennent à usurper sur les habitudes éditoriales en publiant des ouvrages illustrés de leur main. Leurs incursions plastiques ne dépassent pas, le plus souvent, le stade du manuscrit. Ainsi, Hugo garda par-devers lui les dessins de 1866 relatifs aux Travailleurs de la mer (fig. 1), et, plus avant dans le siècle, Clemens Brentano crayonna en 1838 une sorte de frontispice pour son conte Gockel, Hinkel, Gackeleia à destination privée ${ }^{1}$. L'édition se révélait autrement plus hospitalière aux propositions littéraires d'artistes tels que le peintre Edward Lear ou le caricaturiste George du Maurier, le premier illustrant en 1846 son recueil $A$ Book of nonsense, le second son

I. Pour les reproductions, voir S. Linarès, Ecrivains artistes, respectivement p. 143 et 74. 


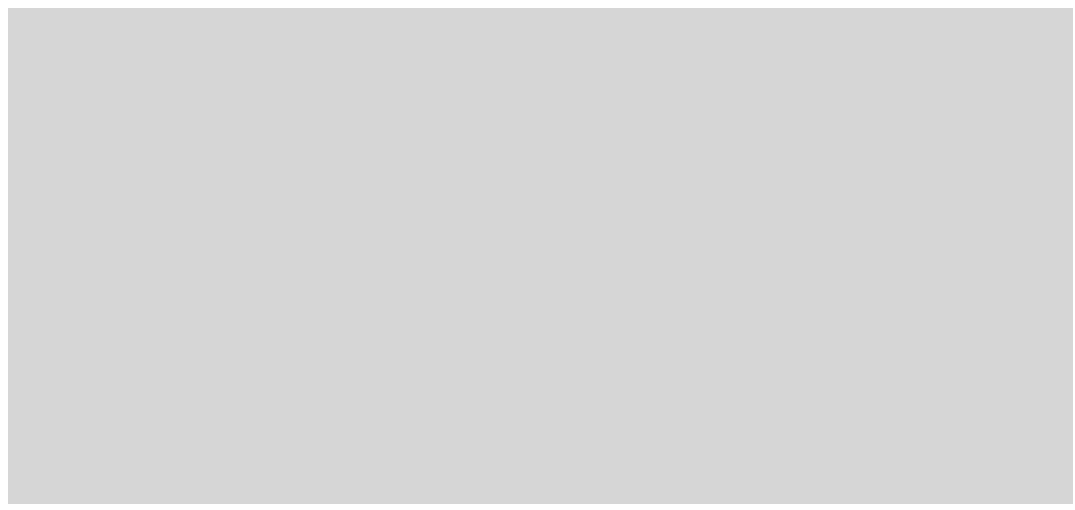

Fig. 2 - Thomas Hardy, In a eweleaze near Weatherbury, illustration pour Wessex Poems and other verses, 1898.

roman Trilby en 1894. On peut supposer que jouait encore, en ces cas, l'attraction pluriséculaire de la peinture pour la littérature, développée depuis la Renaissance afin de revêtir celle-là, antérieurement conçue comme un simple art mécanique, de la dignité d'art libéral que possédait celle-ci. L'échange du pinceau contre la plume disposait d'une légitimité, culturellement construite, qui faisait défaut à la réciproque. Il entrait dans un processus d'élévation esthétique de vieille souche quand la pratique de son contraire menaçait d'égaliser, voire de renverser les conditions ancestrales de l'écrivain et de l'artiste, et de ravaler l'écriture au rang d'activité manuelle - ce contre quoi l'avait toujours prémunie son abstraction native. Toujours est-il que d'aucuns, parmi les auteurs du XIX ${ }^{\mathrm{e}}$ siècle, donnèrent à leurs dessins une existence publique. Par exemple, Thomas Hardy avec la parution de ses Poèmes du Wessex en 1898, pour l'illustration desquels il ne recourut pas à des personnes qualifiées, notamment George du Maurier (son collaborateur graphique pour la publication en revue de ses romans La Main d'Ethelberta en 1875-1876 et Une Laodicéenne en 1880-1881), mais s'en remit à ses propres facultés d'ancien apprenti architecte. Le cas est exceptionnel à plus d'un titre. Hardy met ses images de plusieurs degrés au-dessus du tout-venant du livre illustré. Il les associe à la poésie lyrique, un genre dont la transposition iconique promet moins de facilité que le roman, grand pourvoyeur de scènes et de portraits. Il rend volontiers les rênes à son imagination et franchit les bornes du réalisme, comme dans ce paysage campagnard assorti de lunettes géantes et placé au contact des vers 
intitulés In a eweleaze near Weatherbury ${ }^{2}$ (fig. 2). Certes, l'éventualité de la figuration est fonction du caractère narratif de cette poésie. Toute lyrique qu'elle est, elle fournit en épisodes le désir de représentation de l'auteur. Cependant, la réplique graphique au texte reste souvent à distance de l'évolution relatée, malgré son point d'ancrage. Dans l'illustration susdite, rien du poème n'est reconnaissable à l'exception du site (la région du Dorset). Exprimée au fil des vers, la déploration du poète, en butte aux fuites conjuguées du temps et de l'amour, ne se ressent plus à l'œil nu. L'attachement déclaré à la Beauté est seul à connaître une adaptation visuelle, de l'ordre de la transmutation métaphorique: les lunettes posées à même le paysage objet de leurs regards et face aux yeux du lecteur emblématisent, hors de toute proportion et jusqu'à la réflexivité, l'affirmation verbale du pouvoir contemplatif.

Cette narration transfigurée, conjointement suspendue à la vision de la nature et au spectacle de l'aptitude optique, s'accompagne d'une signature au bas de la composition. Voilà qui relativise sa singularité dans le corpus contemporain des illustrations d'écrivain, souvent portées à jouer de la variation sur le thème de la souscription. L'emplacement des lettres $\mathrm{T}$ et $\mathrm{H}$ dans la continuité spatiale de la barrière, le choix d'un monogramme à l'allure extrême-orientale, tout fait ici dessin. En était-il différemment dans la page de titre du manuscrit des Travailleurs de la mer (fig. 1) ? La mention de l'intitulé coexistait avec la manifestation ouvragée de l'appellation de l'auteur. De fait, il est fréquent que l'écrivain illustrateur garde en réserve de ses images sa fascination pour les formants de l'écriture et qu'il trahisse, sous la figuration des reliefs de ses textes, fussent-ils narratifs, son attrait pour la plasticité scripturale. L'essor du phénomène des écrivains artistes a, pour beaucoup, partie liée avec la contestation d'une appréhension trop phonocentriste du système alphabétique, comme avec l'objection au calibrage standardisé des lettres imprimées. Les Histoires comme ça de Kipling en sont aussi témoin. Ce fut grâce au succès des deux tomes du Livre de la jungle, parus en 1894 et 1895, que Kipling parvint à publier en 1902 ce recueil de contes pour enfants avec des planches de son cru. Comme souvent à l'époque, la destination de la littérature de jeunesse, méjugée en comparaison de la production dite sérieuse, offrait plus de latitude à l'auteur pour exprimer, à la vue de tous, sa veine artistique. Il n'en demeure pas moins que Kipling

2. Ibid., p. 83 . 
Fig. 3 - Rudyard Kipling, La Première lettre, illustration pour Histoires comme ça, London, Macmillan \& Co, 1902.

tenait ici, en mots et en images, tout un propos sur la nature de l'écriture. La question des origines qui réunit l'ensemble de ces textes affecte avec faveur la graphie elle-même, puisque deux contes, "La première lettre» et "Comment s'est fait l'alphabet", mettent en fiction la naissance de la transcription. Kipling y attribue à une petite fille du Néolithique prénommée Taffy l'invention de l'écriture en deux temps: d'abord le dessin figuratif, qui prête aux malentendus d'interprétation, puis son glissement vers la notation phonétique, qui préside à la naissance de 
l'alphabet. Premièrement, l'héroïne grave avec une dent de requin sur un morceau d'écorce de bouleau un message adressé à sa mère sous forme représentative; une semaine après, elle transpose les sons en caractères toujours moins mimétiques sous l'effet de la simplification du tracé. Sur cette lancée, la dimension narrative des contes réunis dans Histoires comme ça, si elle n'est pas interdite de résidence dans les illustrations, se ressent volontiers de l'obsession scripturale que connaît l'auteur. Il suffit, pour s'en convaincre, de considérer la planche jointe au conte intitulé «La première lettre» (fig. 3):

Ceci, nous informe la légende, c'est l'histoire de Taffimai Metallumai [dite Taffy] gravée sur une vieille défense, il y a très longtemps, par les Anciens Peuples ${ }^{3}$.

L'enchaînement des vignettes épouse très exactement le fil du récit, ainsi que le précise le narrateur:

Si tu lis mon histoire, ou si on te la lit, tu verras comment tout cela est raconté sur la défense.

L'image se présente donc comme la copie d'une pièce archéologique, qui reprend la découverte initiale de Taffy, soit le dessin figuratif ${ }^{4}$, et la perfectionne, non seulement grâce au progrès de la maîtrise artistique, mais surtout grâce à la création d'une séquence narrative, découpée en cases. A cette illustration synthétique, qui rassemble les phases de l'histoire, s'ajoute un second plan de représentation, surclassant le premier: sur les côtés de l'image centrale s'accumulent des lignes de runes dont l'extension démesurée et le persistant mystère en imposent à la transparence sémantique de la séquence silhouettée. Deux époques de l'écriture se trouvent ici réunies, mais c'est au bénéfice de la seconde, celle des syllabaires, dont Kipling se garde d'expliciter le sens. "Les lettres autour de la défense sont magiques - des Runes magiques - et si tu pouvais les lire, tu découvrirais quelque chose de plutôt nouveau" 5 , explique-t-il à son jeune lecteur. Qu'est-ce à dire? Non content de suggérer la genèse spirituelle, divinatoire notamment, de l'écriture - évacuée de la fable, peut-être eu égard au jeune lectorat visé -, Kipling,

3. R. Kipling, Histoire comme ça, p. 94.

4. Voir la reproduction dans Ibid., p. 90.

5. Ibid., p. 94. 
Fig. 4 - Rudyard Kipling, Le Commencement des Tatous, illustration pour Histoires comme ça, London, Macmillan \& Co, 1902. 
sans consulter l'exactitude historique ni la vraisemblance de l'intrigue, fait montre, jusqu'à l'évidence visuelle, de sa fascination pour l'iconicité de l'écrit.

Cette fascination se maintient au même diapason dans d'autres illustrations du recueil Histoires comme ça, sans caution fictionnelle aucune. Ainsi, dans "Le léopard et ses taches", qui donne lieu à un portrait de Baviaan, le Babouin, assorti d'écritures de toutes latitudes comme de pure fantaisie, à large portée indicielle ${ }^{6}$. Ainsi dans «Le Papillon qui tapait du pied", où l'effigie du monstre, sorti de la mer, forme le fond sur lequel se détachent les désignations des caisses entreposées ${ }^{7}$. La planche reproduite pour accompagner «Le commencement des Tatous» (fig. 4) lâche plus encore la bride à la plasticité du texte:

Voici, nous explique l'illustrateur, une magnifique carte du turbide Amazone, imprimée tout en noir. Elle n'a rien à faire avec l'histoire, excepté qu'il y a deux Tatous dedans (vers le haut). Ce qu'il y a de beau, ce sont les aventures arrivées aux hommes qui suivirent la route indiquée par les flèches. [...] On commence en bas, au coin gauche, et on suit les petites flèches tout le temps et on revient à la fin, après avoir fait le tour $[\ldots]^{8}$.

Cet appendice narratif au conte principal donne libre champ aux évolutions spatiales et calligraphiques du manuscrit, lequel augmente sa tangibilité à côtoyer des dessins figuratifs. L'itinéraire des explorateurs, débarqués du vaisseau Le Tigre royal, est raconté et imagé tout ensemble en sorte que le rapport illustratif se résorbe dans l'expérience de la fusion des médias et que la circulation du regard d'un état de l'image à l'autre, tantôt verbal, tantôt graphique, fait office d'opérateur de liaison entre les modalités du tracé. Une autre façon pour Kipling de placer ses planches sous la dépendance conjointe de la narration et de l'écriture, de la logique articulée autant que du régime scriptural, c'est de scinder en deux espaces inégaux l'illustration annexée au conte et, selon les cas, de rehausser la part fictionnelle ou la part calligraphique de la représentation. Pour «L'Enfant d'Eléphant», Kipling distribue la surface de

6. Ibid., p. 33 .

7. Ibid., p. 161.

8. Ibid., p. 72. Pour la reproduction, voir p. 73. 
Fig. 5 - Rudyard Kipling, L'Enfant d'Eléphant, illustration pour Histoires comme ça, London, Macmillan \& Co, 1902.

l'image en deux zones déséquilibrées (fig. 5). Celle du haut s'inscrit dans la lignée du récit:

Ceci, c'est l'Enfant d'Eléphant, pendant que le Crocodile lui mange le nez. [...] Il tire très fort, et de même fait le Crocodile; mais le SerpentPython-Bicolore-de-Rocher arrive dare-dare, à la nage, au secours de l'Enfant d'Eléphant ${ }^{\text {. }}$

Quant aux motifs de la prédelle:

Au-dessous du vrai dessin, ce sont des ombres d'animaux africains qui entrent dans une arche africaine. [...] Tout cela ne veut rien dire. Je les ai mis là, parce que j'ai pensé qu'ils faisaient bien.

9. Ibid., p. 46. Pour la reproduction, voir p. 47. 
Fig. 6 - Rudyard Kipling, Le Portrait du Chat qui s'en va par les chemins mouillés $d u$ bois sauvage remuant la queue et tout seul, illustration pour Histoires comme ça, London, Macmillan \& Co, 1902. 
En vérité, la vacuité diégétique de ce complément iconographique est inversement proportionnelle à sa plénitude d'exercice pour l'imagination. Une fois encore, un rêve d'écriture se fait jour: on croirait voir une ligne de pictogrammes et, enfermant l'arche salvatrice, la première lettre de l'alphabet latin. Pour "Le Chat qui s'en va tout seul", Kipling propose un dispositif similaire (fig. 6), à cela près que la partie basse de l'illustration propose un prolongement graphique à la fiction, dont elle constitue un surgeon narratif:

Au-dessous de l'image pour de vrai, il y en a une autre de la Caverne commode où l'Homme et la Femme s'installèrent après la venue du Bébé. [...] L'Homme part sur le dos du Cheval chercher la Vache, afin de la ramener à la Grotte pour se faire traire ${ }^{10}$.

La partie haute, "c'est le portrait du Chat qui s'en va par les Chemins Mouillés du Bois Sauvage, remuant la queue et tout seul». Et le commentateur d'ajouter: "Il n'y a pas autre chose dans l'image, excepté des champignons. " Armé de tous les exemples précédents, on peut en douter. D'abord, le monogramme de l'auteur accroche l'œil à droite de la composition. Et puis, rien n'interdit de déceler dans la perspective des arbres et dans l'amorce de la barre centrale la surimpression de la lettre A, tant Kipling met, dans son ouvrage, d'active volonté à sonder les étapes historiques et les apparences concrètes de l'écriture.

Les Aventures d'Alice au cour de la Terre de Lewis Carroll ouvrent d'autres voies à l'illustration narrative lorsqu'elle est le fait des hommes de lettres, et non des hommes de l'art. Entre 1862 et 1864, son auteur travailla jusqu'au moindre détail à l'élaboration d'une version calligraphiée et imagée par ses soins, dont il espéra en vain la publication jusqu'en 1886, date de sa parution en fac-similé. Face aux réserves de son entourage touchant son talent plastique, Carroll admit provisoirement la nécessité d'une mise aux normes de l'édition contemporaine: ce fut sous une forme imprimée à la presse et illustrée par un professionnel (John Tenniel) que sortit en 1865 la seconde mouture du texte, Les Aventures d'Alice au pays des merveilles. La version primitive de l'œuvre ne trouva rien dans les origines de l'écriture qui occupât son attention, contrairement aux Histoires comme ça. Cependant, la réalité palpable de la graphie appela toute la patience du scripteur à ses manifestations dans

IO. Ibid., p. 146. Pour la reproduction, voir p. 147. 


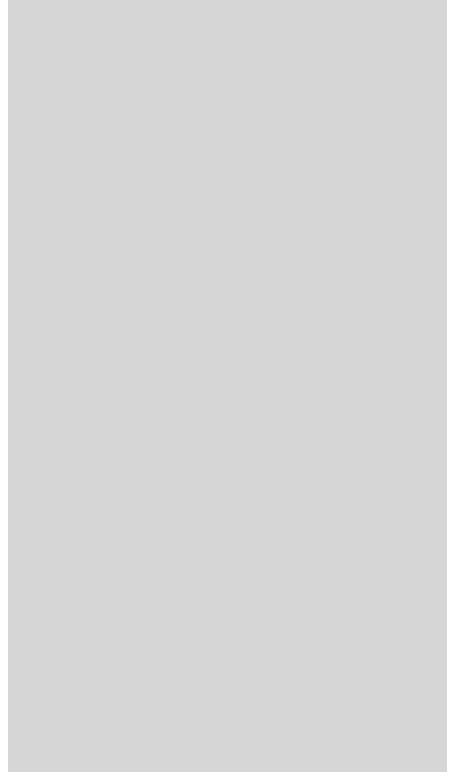

Fig. 7 - Lewis Carroll, Les Aventures d'Alice au cour de la Terre, page de couverture, 1864. le livre. Pour la page de titre comme pour la dédicace et les têtes de chapitre, Carroll recourut aux lettres gothiques, voire à la polychromie, à telle enseigne qu'il paraît moderniser la tradition du manuscrit enluminé ${ }^{11}$ (fig. 7). Le texte lui-même, de loin en loin orné d'images, ne cherche pas a priori l'effet visuel, faute d'expressivité dans la rédaction manuscrite. Les exceptions ressortissent à la parodie des caractères imprimés et suggèrent la nature délibérée de leur abandon dans l'ouvrage (fig. 8) ${ }^{12}$. Le contraste entre le contenu des chapitres, le plus souvent rédigé sans relief, et le paratexte, ouvragé à la façon médiévale, semble volontaire. Selon toute hypothèse, l'accentuation de l'illustration coïncide avec la raréfaction des singularités calligraphiques, tandis que l'intensité du tracement verbal est en raison inverse de la prégnance de la figuration. On pourrait opposer à une telle idée que, à défaut d'illustration véritable, le motif floral s'harmonise au paratexte dans le livre, mais c'est précisément parce qu'il vaut pour déclinaison végétale de l'écriture. Le cœur de l'ouvrage, notamment les pages d'illustrations in-texte, établit à ce point une connivence graphique entre l'inscription des lettres et le dessin des motifs que la bichromie du noir et blanc y règne sans partage (à la réserve de l'image finale, relevée de rouge ${ }^{13}$ ) et qu'elle uniformise pour l'œil les moyens d'expression.

Le rapport de proximité qui lie l'écriture et l'illustration confine même à l'équivalence dans la création d'un calligramme au chapitre II (fig. 9). Car l'histoire de la souris, mêlée dans le souvenir d'Alice à la vision de la queue de l'animal, cause en retour la représentation textuelle

II. L. Carroll, Les Aventures d'Alice au cour de la terre, $2^{\mathrm{e}}$ page de garde.

I2. Ibid., p. 7.

13. Ibid., p. 88. 


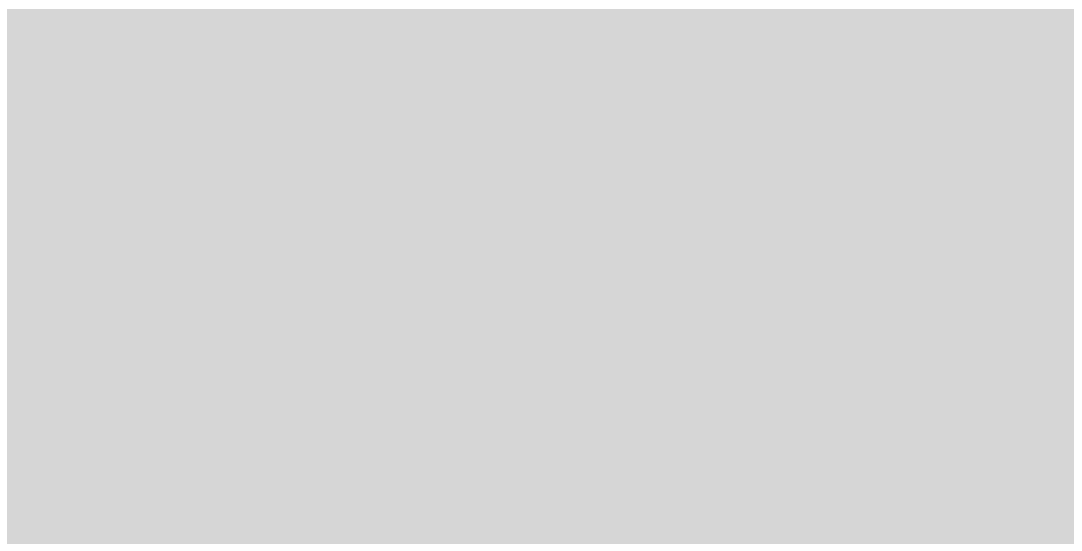

Fig. 8 - Lewis Carroll, Les Aventures d'Alice au cour de la Terre, chapitre 1, folio 7, 1864.

de cet appendice caudal ${ }^{14}$. La parenté que l'on supposait entre la graphie et l'image culmine dans l'identification de l'une à l'autre, puisque l'organisation langagière épouse alors étroitement la représentation graphique. Si le texte et l'illustration ne font plus qu'un, il n'est pourtant pas certain que Carroll tienne pour acquise et légitime une pareille confusion des médias. Au-delà de son aspect ludique, le procédé mimétique repose sur un défaut de compréhension d'Alice qui entend, par calembour, a long tail ("une longue queue») en lieu et place de a long [...] tale ${ }^{15}$ ("une longue histoire») et qui, sur la base de ce malentendu, amalgame l'apparence de la queue de la souris et le fil de sa narration. Cette équivoque plonge Alice dans ses pensées: " "Votre queue est longue, c'est sûr”, dit Alice en abaissant avec étonnement son regard vers la queue de la souris [...]. Et elle continua de se creuser la tête à ce propos, tandis que la souris parlait, si bien que l'idée qu'elle se fit de l'histoire ressembla à ce qui suit», c'est-à-dire au calligramme. En effet, se déroulent alors et les courbes de la queue et les aléas du récit:

Nous vivions en ce temps-là, / Sous le paillasson comme des rois; / Mais un gros malheur arriva, / C'était le chat! / Toutes nos joies / devenues pépins, / Devant nos yeux / plus un point, / De nos espoirs / plus rien, / Ce fut le Chien! / Quand le / chat

I4. Ibid., p. 28.

I5. Ibid., p. 27. 
Fig. 9 - Lewis Carroll, Les Aventures d'Alice au cour de la Terre, chapitre 2, folio 28, 1864. 
est / au loin, / Les souris / font festin, / Mais hélas! / Un jour / funeste, / (comme / on dit), / Vinrent / le chien et / le chat chassant / un rat, / Et / voici / nos / souris / Tout / d'un / coup / toutes aplaties, Sous le paillasson comme des rois, Voyez-vous ça! ${ }^{16}$

L'état de contemplation de l'héroïne, causé par son trouble, autorise seul la communion du visible et du lisible, la concordance des temps du présent et du passé, la concomitance de l'instant plastique et de la durée verbale. En vérité, passé l'immédiateté de la saisie optique du dessin, sa dépendance langagière, sa soumission à l'ordre narratif le font entrer dans une dynamique diachronique qui lui restitue le sens de la temporalité, perdu à la réception, mais vécu à la création. Avec ce calligramme, le lecteur connaît, joint à l'impression rétinienne de l'image, le sentiment d'assister à une illustration en instance de réalisation, subordonnée au délinéament des mots du scripteur comme à la progression du récit que narre la souris. Bien plus, ce qui est en voie de représentation sous nos yeux consiste en une transposition mimétique, non pas d'un élément précis de l'histoire de la souris, mais d'une caractéristique physique de l'énonciatrice. Dans ces conditions, le calligramme ne figure pas, comme attendu dans une illustration traditionnelle, une composante du récit enchâssé dans la fiction en cours, mais répercute graphiquement la situation de communication principale. Il dévoile à la fois la nature de la narratrice et le point de vue d'Alice sur elle, étant en somme la résultante iconotextuelle d'une focalisation interne.

Cette interférence entre l'illustration de la narration et la situation d'énonciation, permise par l'emploi plastique de l'écriture, se manifeste ailleurs dans l'ouvrage, sur le plan du discours d'ensemble, qui place auteur et lecteur dans un même contexte. Sans doute n'y a-t-il pas de calligramme qui soit directement à la charge du narrateur et à l'adresse du lecteur. Il n'en demeure pas moins que Carroll procède à des aménagements spatiaux qui exhibent l'objet livre lui-même et, par là, instaurent des effets de spécularité. Par exemple, la récitation du poème "Vous êtes vieux, Père Guillaume» s'accompagne de quatre illustrations qui concordent thématiquement avec le récit dialogué fait par Alice ${ }^{17}$ (fig. 10). Les choix iconographiques sont alors déterminés par les

I6. Ibid., p. 28.

17. Ibid., p. 52 sq. 


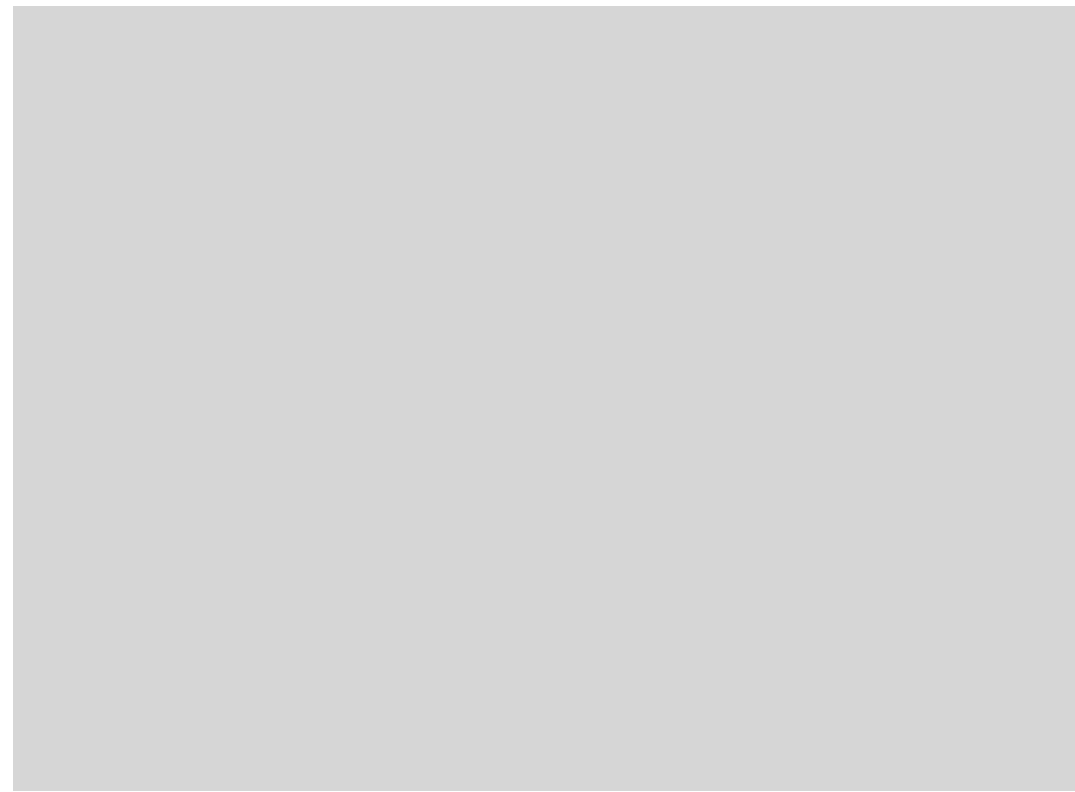

Fig. 10 - Lewis Carroll, Les Aventures d'Alice au cour de la Terre, chapitre 3, folios 52-53, 1864.

orientations fictionnelles du poème, en conformité avec l'usage courant. Mais un autre niveau de lecture, préalable et supérieur au précédent, affecte sans médiation la relation du lecteur à l'objet de ses regards: aussi bien la connaissance du texte que la considération de l'image, et donc l'estimation du degré de leur convergence, sont tributaires du maniement du livre, qu'il convient de faire pivoter d'un quart de tour. Or, cet acte est en pleine conformité avec les premiers vers, qui évoquent la position renversée du Père Guillaume:

"Vous êtes vieux, Père Guillaume», dit le jeune à l'aîné, / «Et vos cheveux blancs sont clairsemés / Sur la tête pourtant vous restez planté - / Est-ce, à votre âge, ce que vous devriez?»

Il appert donc que le poème se montre doublement illustré: et par l'image en vis-à-vis, où Père Guillaume fait le poirier, et par la configuration de la double page, que seul le geste tournant du lecteur permet de rétablir à l'endroit. L'emplacement du dessin comme du texte illustre ainsi, au stade suprême, la teneur du propos récité par Alice. 
Sans aller jusqu'à ce point de complexité dans le dispositif iconique, Le Petit Prince (1943) n'est pas exempt d'une dimension réflexive qui révèle une fois encore combien le passage à l'image constitue souvent pour les écrivains une façon de faire retour sur le langage et d'en poursuivre l'iconicité. Certes, Saint-Exupéry ne met pas l'accord entre les systèmes plastique et linguistique en publiant son écriture en reproduction de manuscrit. Il n'offre pas plus à l'imprimé l'opportunité de devenir graphique à la faveur de compositions calligrammatiques. Il recourt tout au plus à l'illustration et, sous couvert d'une simple mise en images de son récit, exprime la tentation scopique qui actionne les ressorts de son expression verbale. Le Petit Prince dissimule, sous le récit de la rencontre du narrateur avec le héros éponyme, une autre histoire, moins fictionnelle: le dépassement du traumatisme graphique de l'auteur, noué dès l'âge de six ans. Le chapitre I relate comment il fut contraint de renoncer à "une magnifique carrière de peintre» face à l'incompréhension des adultes, incapables de reconnaître les motifs de ses deux premiers dessins (le boa vu du dehors, puis le même en coupe). Dans le chapitre suivant, le Petit Prince, outre qu'il se révèle le seul apte à identifier le «boa fermé» ${ }^{18}$, se satisfait des maigres aptitudes artistiques du narrateur, qui répond à ses instances en lui dessinant plusieurs moutons. Autant dire que la présence d'illustrations dans l'ouvrage manifeste la portée curative de l'aventure relatée. La fiction porte en sous-œuvre une autre matière à récit: le retour de l'auteur à l'esprit d'enfance et à l'acte graphique. Au surplus, cet acte, s'il ne recouvre visuellement que des implications figuratives, pourrait bien avoir pour secrète origine le désir de signifiants motivés, à tout le moins saturés de réalité. A l'appui de cette hypothèse, on comparera les deux dernières images du livre, qui reconduisent le même paysage de désert surmonté d'une étoile ${ }^{19}$. De l'une à l'autre, le lecteur assiste, non seulement au trépas du héros, piqué à mort par le serpent, mais à l'effacement d'un astre, ce Petit Prince dont la chevelure blonde, vue de dos, ne s'apparente jamais mieux à une étoile que dans la représentation de sa chute. Du reste, dès son premier portrait, la parenté du personnage avec les astres est suggérée par ses épaulettes constellées ${ }^{20}$; effigie après effigie, son écharpe, curieusement dressée,

I8. A. de Saint-Exupéry, Le Petit Prince (1945), p. 16.

19. Ibid., p. 94 et 96.

2o. Ibid., p. 15. 
l'apparente à une comète, dotée d'une chevelure et d'une queue ${ }^{21}$. Et ses visites successives à des planètes filent, en la fictionnalisant, la métaphore stellaire qui lui donne corps. A la vision rapprochée, cette image qu'incarne l'enfant blond tombé du ciel est empreinte d'une étymologie qui permet de l'interpréter sous le rapport de l'écriture: le signe, notamment linguistique, a pour racine le mot signum, "astre» en latin. La confusion entre les langues, matrice d'un rapprochement entre les actions de dessiner et d'écrire, transparaît d'ailleurs dans le chapitre XXIV:

Quand nous eûmes marché, des heures, en silence, la nuit tomba, et les étoiles commencèrent de s'éclairer. Je les apercevais en rêve, ayant un peu de fièvre, à cause de ma soif. Les mots du petit prince dansaient dans ma mémoire ${ }^{22}$.

L'observation mentale des astres n'est-elle pas rendue, par juxtaposition, concomitante du souvenir des paroles du héros? Au bout du compte, la reconquête du dessin, explicite dans le livre, témoigne d'une autre quête, inapaisée, mais sans doute prépondérante: celle d'une écriture épiphanique, dotée de la magique puissance de faire advenir ses référents. Lapparition, puis la disparition du petit prince vaudraient pour naissance et mort d'un signe vivant sur l'écran du désert et du ciel.

Cette succession d'exemples, pris dans le vivier des livres illustrés par leurs auteurs, s'est attachée à démontrer que l'appréhension graphique de la narration compose, sous des formes diverses, avec la fascination scripturale des écrivains. Si le fil du récit trouve des réponses figurées dans les images d'accompagnement, il se révèle suspendu par places à une autre logique que celle du suivi diégétique: l'obsession, plus ou moins déclarée, pour la concrétude de l'écriture dont sont interrogées en sous-main les possibilités de raccord avec le monde qu'elle vise à énoncer. On aurait beau jeu de développer une pareille interprétation à l'égard des histoires mixtes, qui resserrent fortement les liens entre ces modalités du visible que sont le vecteur iconique et le canal langagier. On préférera insister dorénavant sur la fonction identitaire dont ces œuvres peuvent être revêtues à des degrés distincts. Après avoir envisagé le livre illustré d'auteur comme un point d'attraction pour l'imaginaire scriptural, audelà de l'apparente divergence des médias sollicités, on mettra l'accent

2I. Ibid., p. 87.

22. Ibid., p. 79. 
sur la valeur autocentrée de certaines histoires mixtes. Dans l'un et l'autre cas, le moi de l'écrivain trouve à questionner sa nature: là le statut de l'écriture, ici la formation du sujet, étant entendu que la prospection de l'iconicité du langage touche aussi à la substance de la personnalité créatrice.

Musset est de ceux qui ont produit, en écrivains, des albums graphiques, légendés de leur main. Sa position dans le corpus considéré intéresse d'autant plus que les ouvres de cette veine (Le Mariage de Pauline Garcia avec Louis Viardot, 1840; Le Voyage en Sicile, 1843) sont d'inspiration légère, pour tout dire humoristique, et, à l'instar des œuvres pour la jeunesse, ne paraissent pas devoir loger une interrogation de fond. Prenons le premier ensemble, composé de 51 dessins dont 44 sont attribués à Musset, les autres au sculpteur Jean-Auguste Barre. Il relate les péripéties du projet de mariage, ponctué de ruptures, de la cantatrice Pauline Garcia avec le directeur du théâtre des Italiens, lequel apparaît finalement en rival heureux des deux illustrateurs. D'où la charge dont Louis Viardot est victime: son nez disgracieux devient la cause des aléas de cette union et l'objet de métamorphoses caricaturales $^{23}$ (fig. 11). Musset lui-même se représente sous un jour peu flatteur: la chronique nous le montre "malade d'amour [pour Pauline Garcia] et d'une fluxion de poitrine», selon le sixième feuillet (fig. 12). Comme cette série fut réalisée durant la convalescence du poète, la critique s'accorde à lui attribuer des vertus exutoires. Toujours est-il que l'état de crise vécu par Musset trouva une issue dans la représentation plastique de soi, si moqueuse fût-elle. Pour secondaire quapparaisse le personnage de l'auteur dans cette histoire, en comparaison de l'importance accordée aux futurs mariés, voire à George Sand (qui joue les conciliatrices), il semble connaître ici une phase décisive de son parcours biographique: il récupère son assise, retourne au foyer de son individualité éprouvée par le mal physique et par la souffrance sentimentale, à la faveur de la projection iconique de soi, qui le dissocie, le temps de la création, de sa personne, vue par lui du dehors et à distance ironique. Une forme de dédoublement opère alors qui subordonne la reconquête intérieure à la capacité de détachement et d'objectivation de soi dans l'image. De fait, le dénouement de l'album signe la réussite de l'entreprise: le dernier feuillet

23. Pour la reproduction de la série graphique, voir S. Linarès, Ecrivains artistes, p. 88 sq. 
Fig. 11 - Alfred de Musset, Le Mariage de Pauline Garcia avec Louis Viardot, planche $14,1840$.

(fig. 13) nous présente sans raillerie Musset à sa table de travail, "guéri de sa fluxion", et qui, revenu à son état de poète, "fait un sonnet pour une inconnue». Le choix du véhicule plastique entre également dans ce processus d'extériorisation: exécuté en amateur, le dessin s'inscrit délibérément en mineur dans l'œuvre de Musset, et n'entend pas témoigner de sa créativité profonde, d'essence verbale. Il permet de déporter le sujet lyrique vers la périphérie de ses puissances d'invention, et de lui ouvrir l'accès à une altérité curative. En définitive, le manque de vocation pour l'art graphique ouvre à Musset une échappée salvatrice hors du champ verbal, le temps nécessaire de la refonte identitaire. L'omniprésence des légendes lui permet pourtant de ne pas rompre net avec ses dispositions littéraires et de préparer son retour à l'expression articulée.

La problématique du double se vérifie sur d'autres plans dans cette histoire mixte, tant il est vrai que la duplication du moi, pour peu qu'elle soit inexacte et différentielle, promet une stabilité à la personnalité de Musset, intérieurement tourmentée. La fraternité est, chez lui, un facteur d'équilibre. Dans Le Voyage en Sicile, il relate les aventures de son frère en Italie comme celles d'un autre lui-même, optant pour le patronyme commun afin de désigner son héros et s'insinuant graphiquement dans les interstices du récit que Paul écrivit de son côté et qu'il publia 
Fig. 12 - Alfred de Musset, Le Mariage de Pauline Garcia avec Louis Viardot, planche 11, 1840.

Fig. 13 - Alfred de Musset, Le Mariage de Pauline Garcia avec Louis Viardot, planche 17, 1840. 
en 1845 sous l'intitulé Course en Voiturin. Dans Le Mariage de Pauline Garcia avec Louis Viardot, il établit un rapport de confraternité avec le sculpteur Barre à plusieurs niveaux. C'est d'un commun accord que les deux hommes réalisent cette œuvre. La bipartition sémiotique des langages convoqués dans l'album se complique d'une division du travail créateur qui ne coïncide pas absolument avec les spécialités de chacun des collaborateurs (Musset se faisant tour à tour dessinateur et légendier). De surcroît, l'histoire ne place pas seulement Musset et Barre dans une situation amoureuse équivalente (le duo des rivaux malheureux); convertie en narration graphique, elle donne lieu à une syntaxe visuelle qui confirme la parenté symbolique. Séparés dans la diégèse, ils n’en apparaissent pas moins toujours dans les mêmes feuillets, case contre case $^{24}$, avant la dernière image qui instruit un parallèle iconographique entre leurs destins (fig. 13).

Plus encore chez Cocteau que chez Musset, l'histoire dessinée est sujette à l'émergence d'une "identité narrative» ${ }^{25}$ (Paul Ricœur) qui tiendrait du croisement du visuel et du textuel. A l'origine du revirement poétique de Cocteau en 1913, qui le conduisit à renier ses publications antérieures, il y eut une image: l'Eugène ${ }^{26}$ (fig. 14), laquelle ouvrit une campagne de dessins destinée à constituer l'«Album des Eugènes", puis à provoquer l'écriture de légendes, enfin la rédaction d'une autre fiction, Le Potomak. Cette figure, survenue dans le demi-sommeil, prend d'emblée, dans l'esprit de Cocteau, la valeur d'une apparition d'outre-tombe, qui l'entraîne dans un vertige mortifère jusque-là tenu en lisière. L'histoire graphique qui s'ensuit raconte ainsi la palingénésie forcée d'un couple de bourgeois, les Mortimer, attaqué par une escouade d'Eugènes et de femmes Eugènes, qui leur font vainement connaitre le sentiment de finitude par anticipation ${ }^{27}$. Rien qui, selon toute apparence, prenne ici la dimension d'un questionnement identitaire. En vérité, si cette histoire dessinée est pénétrable aux influences des troubles du moi, c'est qu'elle fait fond sur un drame d'enfance, soigneusement camouflé dans la fiction. Toute la

24. Ibid., feuillets 4 et 13 .

25. Voir P. Ricœur, Temps et Récit.

26. Pour la reproduction de l'Eugène, voir J. Cocteau, Euvres romanesques complètes, p. 36.

27. Voir "Album des Eugènes", in J. Cocteau, Euvres romanesques complètes, p. $37-168$. 
Fig. 14 - Jean Cocteau, Calque de l'envoyé des Eugènes et de la première femme Eugène, illustration pour Le Potomak (1913-1914), précédé d'un Prospectus (1916) et suivi des Eugènes de la guerre (1915), Paris, Société littéraire de France, 1919.

figure de l'Eugène est organisée autour d'un œil unique et rond, que l'on peut associer à l'obturateur focal de l'objectif photographique. Or, en 1898, Georges Cocteau se suicida d'une balle dans la tête en laissant à son fils Jean, alors âgé de huit ans, le sentiment que son père s'était rendu coupable d'un parjure à son égard pour ne pas avoir accompli la promesse, faite la veille, de lui rapporter un appareil photographique en réparation. Dès ce jour, mort et photographie unirent leurs destinées dans l'imagination de Cocteau, et leur convoi funèbre fit l'objet de multiples variations dans son œuvre, à commencer par Les Mariés 
de la tour Eiffel, pièce qui prend pour base les égarements d'un appareil de photographie détraqué. Qui plus est, le nom Eugène, donné au premier envoyé de l'invisible, personnalise au plus profond le conte micomique, mi-horrifique qui se déroule, une image après l'autre, dans l'album. Sans doute Cocteau ne se représente-t-il pas lui-même comme Musset, mais sa tragédie psychique n'en gouverne pas moins la désignation de son personnage. Eugène était un de ses prénoms propres et un de ceux de son grand-père Louis-Eugène, figure paternelle de substitution après la mort de son géniteur. Sans doute le terme "ange», en permutant les syllabes du prénom Jean, autorisa-t-il par la suite Cocteau à portraiturer la part infinie de soi par le biais de cette figure spirituelle; mais l'Eugène fut déjà pour lui une façon de se confronter à ses abîmes intérieurs, à ses morts bien aimés comme à sa personnalité perturbée dès l'enfance. L'importance décisive de l'«Album des Eugènes» dans l'œuvre de Cocteau tient précisément à ce qu'il lui a permis de donner un visage à la mort qu'il avait intériorisée, voire refoulée, de cerner d'un contour son malaise psychique, sa "difficulté d'être». Reste que Cocteau adopte tout du long la focalisation zéro pour considérer ses créatures, sans s'impliquer explicitement dans la fiction. Qui plus est, cet album était à l'origine muet. "A la longue, explique Cocteau à l'ouverture, je me reconnus le droit de joindre un texte aux dessins après avoir pensé qu'ils n'en comportaient pas.» ${ }^{28}$ Le dessin fut donc premier à naître sous la plume: premier parce que seul le domaine de l'image, moins conceptualisé, pratiqué dès le plus jeune âge et partagé avec le père, peintre amateur à ses heures, était propice, chez Cocteau, à l'éclosion inconsciente de son traumatisme. Le langage, quant à lui, fut second: il vint ordonner et clarifier la fiction pour qu'elle échappât à la simple fonction d'exorcisme des démons intérieurs. Un besoin de compréhension se fit sentir dans la sensibilité de Cocteau et, avec lui, ce fut la littérature qui reprit le dessus sur l'image par laquelle elle était hantée. Aussi Cocteau, après avoir estimé que Le Potomak, qui contenait l'"Album des Eugènes", avait rang de préface à son œuvre entière, le fit-il entrer dans la catégorie "roman» de sa production poétique. La conjonction du dessin et du discours ne lui avait-elle pas dispensé la possibilité de revenir sur son roman familial et de fixer son "identité narrative», grâce à l'investissement du sujet individuel dans la fable et

28. Ibid., p. 39. 


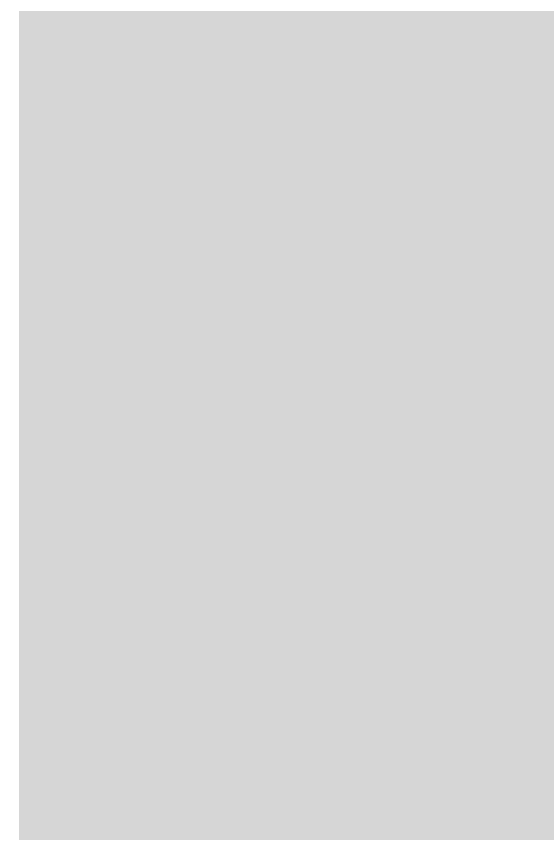

Fig. 15 - Franz Kafka, Sans titre, croquis extraits d'un carnet de lecture, 1905. grâce au besoin d'intelligibilité du vécu personnel?

Comme nous le prouve l'exemple de Cocteau, les histoires mixtes ne paraissent souvent doubles que sous l'angle sémiotique, et peuvent converger vers une même tentative d'élucidation de l'intériorité. Les études de cas ici réunies ont eu pour finalité de démontrer, avec des accents divers, le souci d'unicité qui présidait aux expériences graphiques des écrivains choisis, qu'ils fussent illustrateurs de leurs récits, ou auteurs de narrations visuelles. Imager des fictions, c'est, au vu de nos échantillons, s'enquérir de la portée iconique de l'écriture; raconter des images, c'est faire porter l'interrogation sur le relateur. Les croisements sont évidemment possibles. Sans plus épiloguer, nous terminerons sur un cas limite, qui fusionne le dessin et l'écriture pour les renvoyer à une égale vanité. Dans ces croquis de Kafka (fig. 15), extraits d'un carnet de lecture de 1905, l'histoire dessinée semble orpheline du langage et propose un enchaînement discontinu de six figures noires, dont on peut raisonnablement supposer qu'elles offrent six états d'un même personnage ${ }^{29}$. Les quatre silhouettes centrales combinent en chiasme le motif de l'expectative et le motif de l'abattement. Quant à l'escrimeur pugnace du haut, il trouve son reflet désorienté dans l'aveugle du bas. Une vision de l'existence en somme, dysphorique et désaccordée. Labsence de légendes accentue le défaut de sens dont est ici frappée la vie de tout un chacun. Incarné par un homme reproductible et dépersonnalisé, le sujet perd toute possibilité d'identité. Mais cette figure noire sur fond clair, dans ses poses rigides, ne représente-t-elle pas elle-même un

29. Pour la reproduction du dessin de Kafka, voir S. Linarès, Ecrivains artistes, p. 87. 
ensemble de lettres inarticulées, d'idéogrammes hypothétiques? A croire que l'écriture et la figuration sont alors confondues pour exposer aux yeux la vacuité contemporaine des signes, qu'ils soient nés de la plume ou du pinceau.

Serge LinARÈs Université de Versailles - St-Quentin-en-Yvelines 


\section{BIBLIOGRAPHIE}

Carroll, Lewis, Les Aventures d'Alice au cour de la terre, édition bilingue, Bruxelles, Frémok, 2006.

Cocteau, Jean, Euvres romanesques complètes, édition de Serge Linarès, préface d'Henri Godard, Paris, Gallimard, 2006 (Bibliothèque de la Pléiade).

KIPLING, Rudyard, Histoire comme ça, traduction de Robert d'Humières et Louis Fabulet, Paris, Delagrave, rééd. 1991.

Linarès, Serge, Ecrivains artistes. La tentation plastique (XVIII $-X X I^{e}$ siècle), Paris, Citadelles \& Mazenod, 2010.

Ricceur, Paul, Temps et Récit, tome III : Le temps raconté, Paris, Editions du Seuil, 1985.

Saint-Exupéry, Antoine de, Le Petit Prince (1945), avec des aquarelles de l'auteur, Paris, Gallimard, 2006.

\section{Crédits iconographiques}

Fig. 1 :

Plume, pinceau, encre brune, lavis, gouaches blanche et rouge, aquarelle verte, réserves, sur papier d'album, monté sur la page de titre du manuscrit des Travailleurs de la mer, 19,2 x 25,4 cm, Paris, Bibliothèque nationale de France, département des manuscrits.

Fig. 2:

Encre sur papier, 10,8 x 20,3 cm, Birmingham Museums and Art Gallery.

Fig. 3-6:

Livre imprimé, in- $8^{\circ}$, Londres, British Library.

Fig. 7-10:

Encre sur papier, 18,7 x $11,5 \mathrm{~cm}$, Londres, British Library, département des manuscrits. 
Fig. 11-13:

Mine de plomb et encre sur papier, 20,2 x 30,5 cm, Paris, Bibliothèque de l'Institut.

Fig. 14:

Livre imprimé, in- $8^{\circ}$, Paris, Bibliothèque nationale de France.

Fig. 15:

Encre sur papier, Berlin, Archiv Klaus Wagenbach. 
